\title{
No-Reference Digital Image Quality Assessment Based on Structure Similarity
}

\author{
Basma AHMED ${ }^{1}$, Mohamed ABDEL-NASSER ${ }^{2,3}$, Osama A. OMER ${ }^{3}$, \\ Amal RASHED ${ }^{1}$ and Domenec Puig ${ }^{2}$ \\ ${ }^{1}$ Faculty of Computers and Information, South Valley University, Qena, Egypt \\ ${ }^{2}$ Computer Engineering and Mathematics Department, University Rovira i Virgili, \\ Tarragona, Spain \\ ${ }^{3}$ Electrical Engineering Department, Aswan University, Aswan, Egypt
}

\begin{abstract}
Blind or non-referential image quality assessment (NR-IQA) indicates the problem of evaluating the visual quality of an image without any reference, Therefore, the need to develop a new measure that does not depend on the reference pristine image. This paper presents a NR-IQA method based on restoration scheme and a structural similarity index measure (SSIM). Specifically, we use blind restoration schemes for blurred images by reblurring the blurred image and then we use it as a reference image. Finally, we use the SSIM as a full reference metric. The experiments performed on standard test images as well as medical images. The results demonstrated that our results using a structural similarity index measure are better than other methods such as spectral kurtosisbased method.
\end{abstract}

Keywords. Blind image quality assessment (BIQA), deblurring, point spread function (PSF), structural similarity index measure (SSIM).

\section{Introduction}

Image quality assessment (IQA) is important for numerous image processing measures, such as re-extraction, restoration, enhancement, compression, and acquisition [1]. IQA is divided into Non-reference NR IQA that's refer to the automatic evaluation of image quality using an algorithm so that the only information the algorithm receives before it predicts quality is the distorted image whose quality is obtained, Full-reference FR IQA that requires as input not only the distorted image, but also pristine image which the distorted image quality is evaluated, reduced reference (RR) approaches that possess some information regarding the reference image, but not the actual reference image itself, regardless of the distorted image [11].

Images are affected by different types of distortion during transmission and processing. Therefore, their quality must be addressed or evaluated before they are used. Image quality evaluation is used in various applications such as enhancement, recovery, compression, acquisition, etc. Motion blur is one of the most common artifacts in digital photography [2] [3]. When taking a photo in dim light with a portable camera, tilting the photographer's hand to shake can blur the image. In response to this problem, image deblurring has become an active topic in computational photography and image processing in recent years [6].

The images are blurred due to many reasons such as defects in capturing pictures, low intensity during camera exposure, atmospheric problems, lens defocus, etc. Human 
visual systems are good at being aware of it. But the mechanism of this processing is not fully understood. Therefore, it is difficult to come up with metrics to estimate blur in images. In digital images, there are 3 types of blur effects: average blur, gaussian blur, motion blur. Recently, efforts have been made to develop such blind IQMs devices including spectral kurtosis [4] [13], Blind Referenceless Image Spatial Quality Evaluator (BRISQUE) [16], and the Natural Image Quality Evaluator (NIQE) [17], etc. Some of these IQMs rely on the statistical properties of the deblurred image while others rely on measuring the image quality by basing them on the Human Visual System (HVS)[14][15][20]. Min et al.[6] proposed a blind image quality assessment based on a pseudo reference image and they are using "reference" called pseudo reference image (PRI), and a PRI based blind IQA (BIQA) framework, they develop specific measures of distortion to estimate blockiness, sharpness, and noisiness. PRIbased scales calculate the similarity between distorted image structures and PRI structures. Through a two-stage quality regression after the distortion identification framework, they then incorporate metrics for PRI-based distortion into a generalpurpose BIQA method called the PRI-based blind scale (BPRI). Moreover, BPRI not only performs well in landscape images, but also applies to screen content images. Moorthy and Bovik [15] proposed a blind image quality assessment (IQA) based on the hypothesis that landscapes possess certain statistical properties that are altered in the presence of distortion, rendering them unnatural; and that by characterizing this anomaly using Scene Statistics. Accordingly, they presented an (NR)/blind Algorithm, the distortion identification-based image verity and integrity evaluation (DIIVINE) index, that evaluates the quality of the distorted image without the need for a pristine image. DIIVINE is based on a two-stage framework that includes distortion identification followed by assessing the quality of the specified distortion. DIIVINE can evaluate the quality of a distorted image across multiple distortion classes, against most NR IQA algorithms of a distortion-specific nature.

Lin et al. [19] suggested a simple but highly effective full reference IQA method using Visual Saliency (VS). In the suggested image quality assessment (IQA) model, the role of VS is divided into two parts. The first part, Visual Saliency is used as a feature when computing the local quality map of the distorted image. In the second part, when aggregating the Quality Score, VS is used as a weighting function to reflect the local area's importance. The proposed IQA index is called a visual saliency-based index (VSI). The proposed IQA index VSI performs better while maintaining a moderate computational complexity.

Yue and Jiangming [14] suggested a method for deblurring image method based on local edge selection. The local edges are determined by the difference between the bright channel and the dark channel, after that, a new image deblurring model is created including the term Local Edge Alignment. Obtaining a clear image and core blurring is based on alternating iterations, where the clear image is obtained by ADMM.

Arthur and Lionel [8] proposed a deeper look at three recent measures of severity (global phase coherence, sharpness index, and a simplified version of it), All of which a probabilistic sense measure the surprisingly small overall contrast of the image compared to that of some of the associated random phase fields. They display many theoretical connections between these indicators and study their behavior in a general class of fixed random fields. In the end, they suggested an application to idiosyncratic blindness and demonstrated its efficiency in several examples. 
This paper examines the efficiency of some existing blind IQMs for blind image deblurring like spectral kurtosis and compares it with the proposed SSIM metric as a blind image quality measures IQM [9].

\section{Methodology}

Image degradation model can be described by:

$$
g=H * f+n
$$

where $\mathrm{g}$ is the blurry image, $\mathrm{H}$ indicates the distortion facto, known as the point spread function (PSF), $\mathrm{n}$ is added noise, and * represents the convolution operator [8]. In the spatial field, the PSF characterizes the rate at which the optical system blurs the spotlight [1][10], Figure1 illustrates image deblurring model.

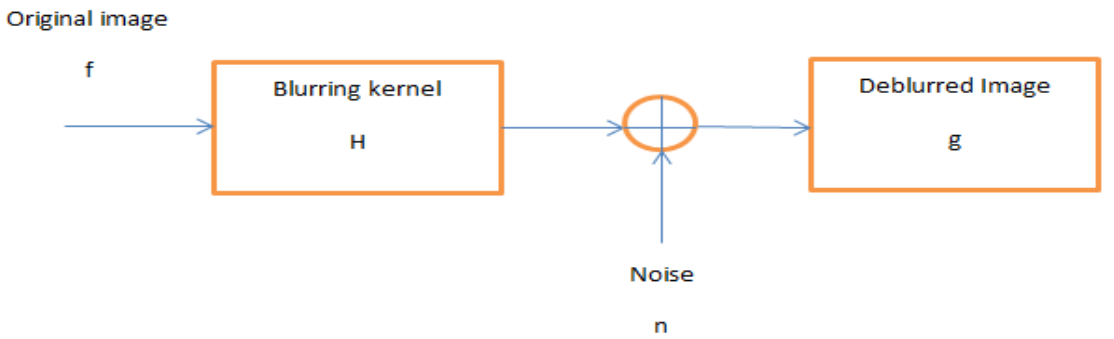

Figure 1. Deblurring image.

In the proposed algorithm a blurred image is obtained by convolving the original image and blurring kernel (PSF). The PSF parameters (angle and length) can be calculated by first estimating the angle quite accurately using analysis in the Cepstrum domain [12], for a given angle we can estimate the length of the blur in the image for a given angle [1]. The Winner filter is applied to the blurred image to get the true image as the equation described below:

$$
G(u, v)=\frac{H^{*}(m, n)}{|H(m, n)|^{2}+N S R}
$$

where $\mathrm{H}$ is the blurring filter and NSR is the noise variance. When the filtered PSF is similar to the real PSF (h) it will be able to reproduce the same blur in reblurred image the restoration filter will produce less noise and resonance. SSIM (structural similarity index measure) is measured for blurred [18], reblurred image to emphasize similar blur reproduction [5][6]. The next pseudocode presents the steps of the proposed reblurring algorithm.

Algorithm1: Pseudocode for the proposed reblurring algorithm.

$1:$ Input pristine image

2: Determine the blurred image by convolving the original image and blurring kernel (PSF) using equation (1) The PSF parameters (angle and length) can be calculated using analysis in the Cepstrum domain 
3: Determine the deblurring image using Winner filter as equation (2)

4: The image has been retrieved by a candidate blurring kernel (PSF)

5: Using SSIM to measure the similarity between blurred and reblurred images.

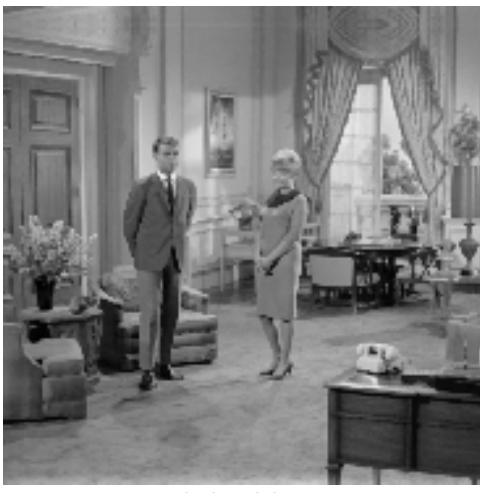

a) Original image

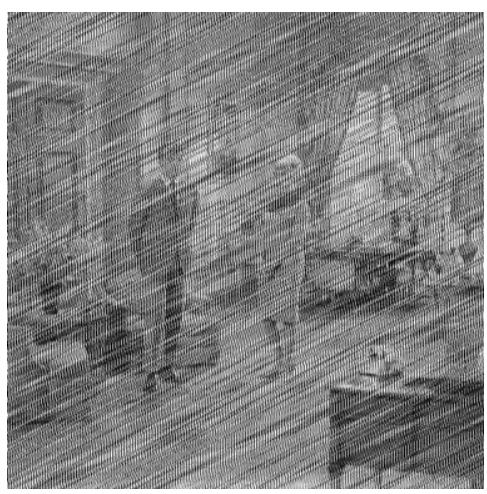

c) deblurred image with an angle

$25^{\circ}$

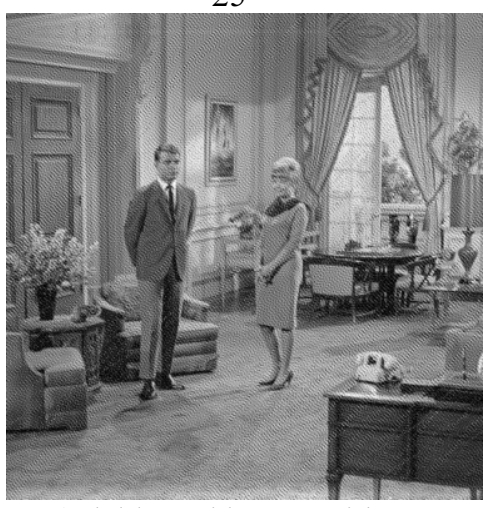

e) deblurred image with an angle $46^{\circ}$

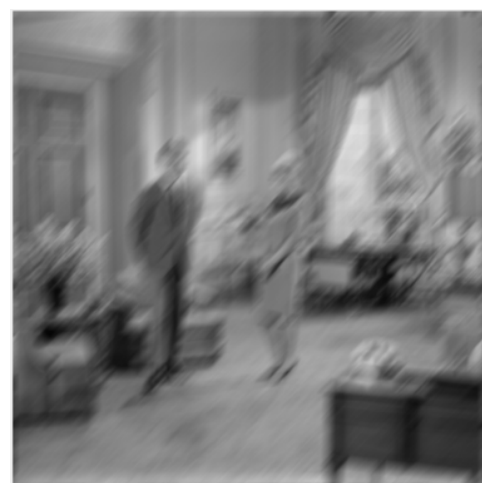

b) Blurred image with an angle $46^{\circ}$

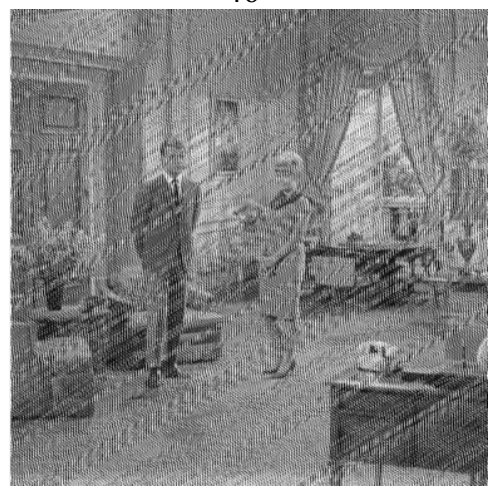

d) deblurred image with an angle $36^{\circ}$

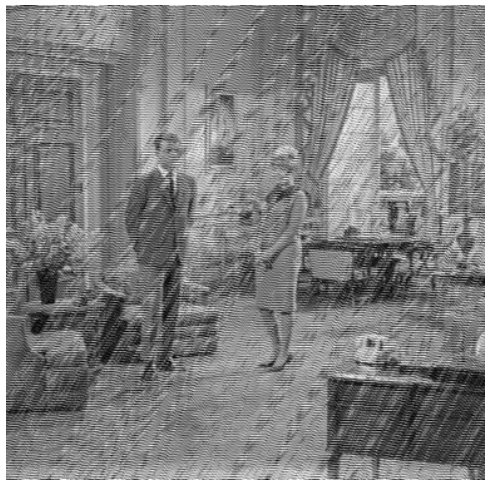

f) deblurred image with an angle $57^{\circ}$

Figure 2. Examples for blind deblurring schema 
An example to illustrate a blind deblurring method is shown in Figure 2(a) the original livingroom image. Figure 2(b) shown livingroom image which was blurred with motion PSF at an angle of 46 degrees. Figure 2(c) and (d)(e)(f) show the Deblurred images due to PSF angle 25 and, 36,46, 57 degrees respectively. Figure 2(e) shows The deblurred image is similar to the original image and the noise level and ringing are tolerated. The image deblurred with a blur kernel similar to the true PSF shown in is the one that will reproduce a similar blur when reblurred.

\section{Experimental Results and Discussion}

Deblurring results using the scheme for images are presented, these include images under motion blur. Experiments for The proposed algorithm include testing of "Standard" test images (a set of images found frequently in the literature: Lena, peppers, cameraman, pirate, etc., all in uncompressed tif format and of the same $512 \mathrm{x}$ 512 size, and some medical images (Eye Fundus image, CT image, etc) as shown in Figure 3.
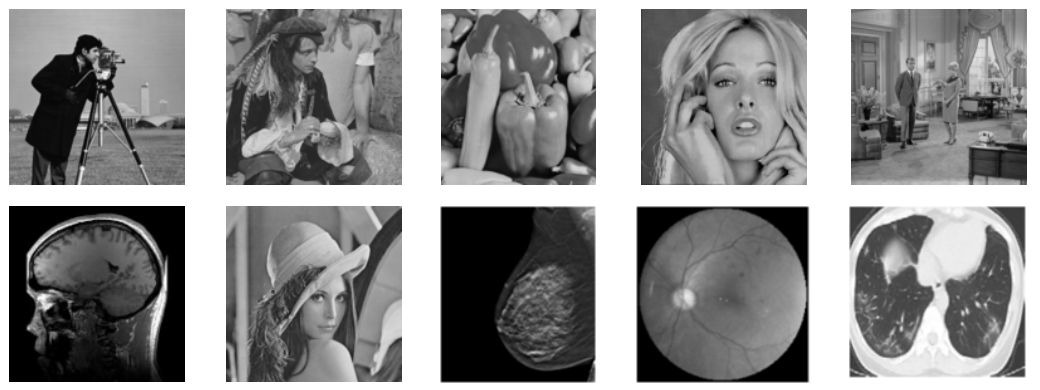

Figure 3. Example of test images

Table 1 summarizes the SSIM results for the blurred. In this case, motion blurred images were used and the PSF parameters angle, theta, was estimated using Cepstrum analysis. From the results shown in Table 1, we find the estimated values close to the theta values used to blur images. SSIM has been calculated for three sets of images: blurred and reblurred, original and blurred, and original and deblurred. The original blurred image and the reblurred image are compared using SSIM and shown by the blurred-reblurred pair column in Table 1. The high values of SSIM depict the reblurred images are in a close approximation of the original blurred images. Therefore the reblurring can estimate the blurring PSFs in the case of motion-blurred images. A higher value of SSIM shows an image of high quality.

In this paper, SSIM were scrutinized. The reblurred images is obtained from reconvolved the deblurred images with the estimated PSF. It turns out that if the candidate PSF is close to the original PSF, it will produce the same blurring in the reblurred image as the original blurred image. As it is evident SSIM based deblurred images produced better visual quality, in the case of motion deblurring. 


\begin{tabular}{lllllll}
\hline \multirow{2}{*}{$\begin{array}{l}\text { Image } \\
\text { Length }\end{array}$} & $\begin{array}{l}\text { Lening } \\
\text { Cepstrum } \\
\text { analysis }\end{array}$ & $\begin{array}{l}\text { Original } \\
\text { Angle }\end{array}$ & $\begin{array}{l}\text { Estimated } \\
\text { Angle }\end{array}$ & $\begin{array}{l}\text { Blurred } \\
\text { Reblurred }\end{array}$ & $\begin{array}{l}\text { Original } \\
\text { Blurred }\end{array}$ & $\begin{array}{l}\text { Original } \\
\text { Deblurred }\end{array}$ \\
\hline livingroom & 23 & 46 & 46 & 0.8936 & 0.4759 & 0.5187 \\
\hline pirate & 21 & 44 & 44.1 & 0.8572 & 0.4836 & 0.5441 \\
\hline woman blonde & 14 & 23 & 23.2 & 0.9064 & 0.6113 & 0.6474 \\
\hline Mandrill & 31 & 46 & 46.5 & 0.9344 & 0.7948 & 0.8104 \\
\hline Camera man & 17 & 33 & 33.2 & 0.8456 & 0.5114 & 0.5571 \\
\hline Goldhill & 23 & 27 & 26.9 & 0.8716 & 0.4177 & 0.4733 \\
\hline Lena & 25 & 13 & 13 & 0.9130 & 0.4747 & 0.5303 \\
\hline Peppers & 20 & 42 & 41.9 & 0.8442 & 0.4004 & 0.5921 \\
\hline Eye Fundus Image & 31 & 41 & 40.3 & 0.9525 & 0.8887 & 0.8964 \\
\hline Tomosynthesis & 23 & 26 & 26.1 & 0.9743 & 0.8658 & 0.8677 \\
\hline CT image & 11 & 32 & 32.2 & 0.7826 & 0.4298 & 0.4910 \\
\hline MRI & 13 & 45 & 45.8 & 0.7050 & 0.5105 & 0.5781 \\
\hline
\end{tabular}

Table 1. SSIM results for the test images

\section{A comparison between the proposed algorithm and spectral kurtosis for image quality assessment.}

By applying the comparison between the proposed scheme and spectral kurtosis method on some images such as (a) corn, (b) boat, (c) barbara, (d) woman darkhair as in Figure 4 with blur angle of 26.5,29, 32.35,47 degrees respectively. Our proposed scheme SSIM error measure estimated the angle as 26.40, 29.1, 32.5, 46.8degrees respectively while spectral kurtosis estimated 26.38,29.3,31.9,49.6 as the blur angle. The corresponding SSIM, spectral kurtosis plot for deblurring images is also shown in Figure 5. We deduce from the shown graph that the SSIM measure maximizes the near the perimeter of the true blurring parameter value. This confirms that our results using a structural similarity index measure are better than spectral kurtosis method.

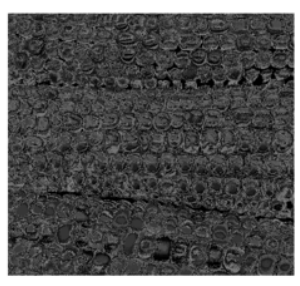

(a)

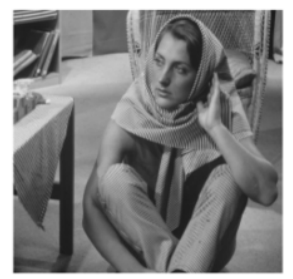

(c)

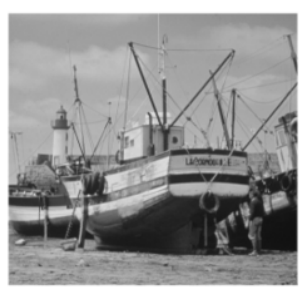

(b)

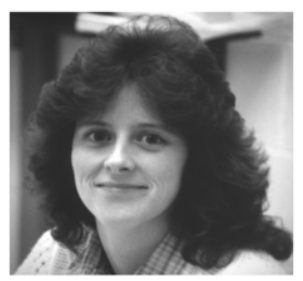

(d)

Figure 4. Examples of images(a)-(d) 


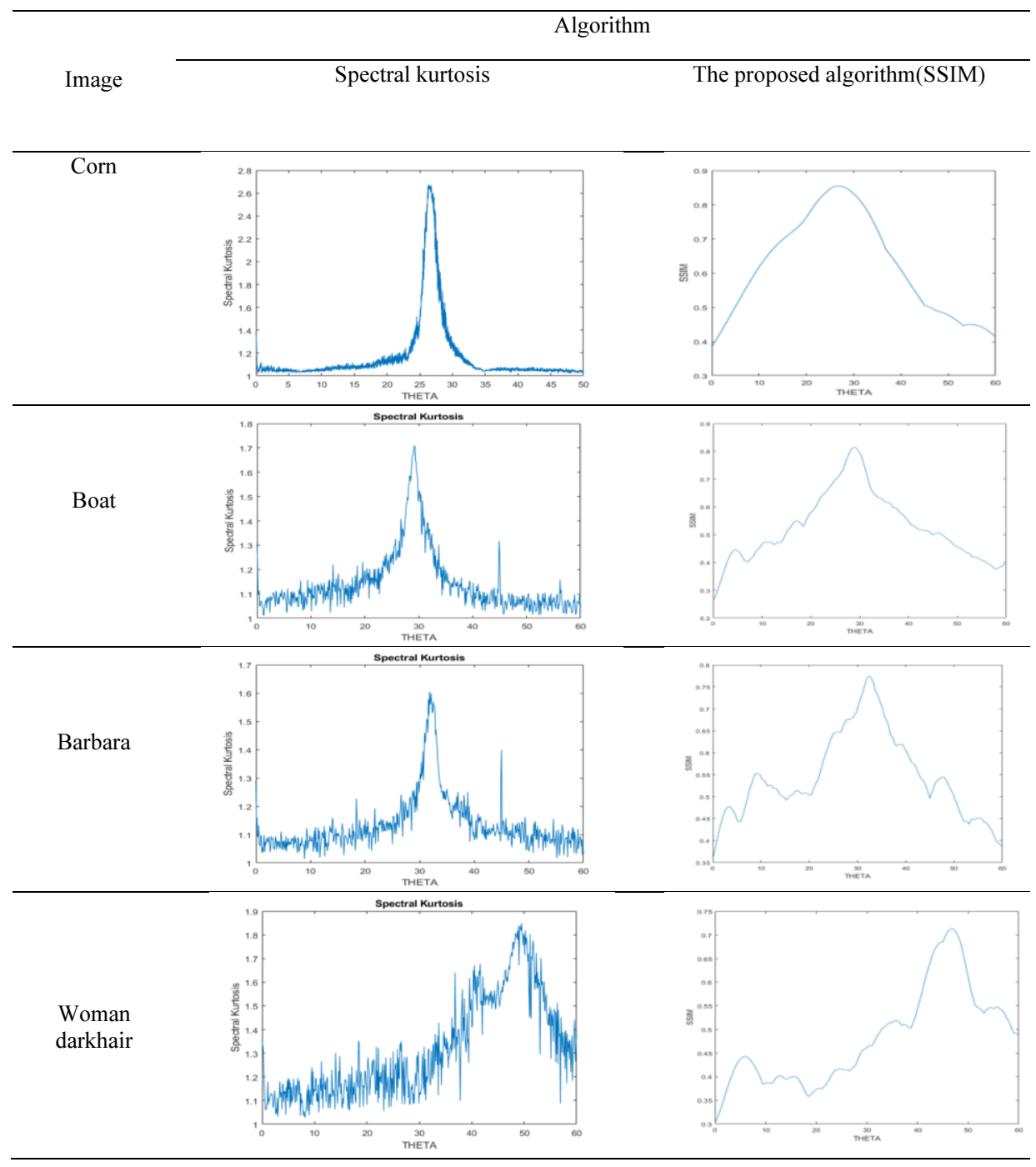

Figure 5. The corresponding SSIM, spectral kurtosis plot for deblurring images

\section{Conclusions}

In this paper, a full-reference blind quality measure SSIM was proposed as a deblurring measure. The proposed SSIM performs well in the case of deblurring by motionblurred images. The proposed SSIM has been rigorously tested and compared with the other state-of-the-art IQA indices like spectral kurtosis on different images such as Standard" test images and some medical images. The results demonstrated that the proposed IQA index SSIM could yield much better-quality deblurring images as compared to others measures such as spectral kurtosis. In future work, for Blind Image Quality Measures (IQMs), it may be interesting to using other metrics and developing them. 


\section{Acknowledgment}

The Spanish Government partly supported this research through Project PID2019105789RBI00.

\section{References}

[1] Chang C-F, Wu J-L, Tsai T-Y. A single image deblurring algorithm for nonuniform motion blur using uniform defocus map estimation. Mathematical Problems in Engineering. 2017.

[2] Almeida MS, Figueiredo MA. Parameter estimation for blind and non-blind deblurring using residual whiteness measures. IEEE Transactions on Image Processing. 2013;22(7):2751-63.

[3] Ahmed IT, Der CS, Hammad BT. RECENT APPROACHES ON NO-REFERENCE IMAGE QUALITY ASSESSMENT FOR CONTRAST DISTORTION IMAGES WITH MULTISCALE GEOMETRIC ANALYSIS TRANSFORMS: A SURVEY. Journal of Theoretical \& Applied Information Technology. 2017;95(3).

[4] Smith WA, Fan Z, Peng Z, Li H, Randall RB. Optimised Spectral Kurtosis for bearing diagnostics under electromagnetic interference. Mechanical Systems and Signal Processing. 2016;75:371-94.

[5] Khan A, Yin H. Efficient blind image deconvolution using spectral non-Gaussianity. Integrated Computer-Aided Engineering. 2012;19(4):331-40.

[6] Min X, Gu K, Zhai G, Liu J, Yang X, Chen CW. Blind quality assessment based on pseudo-reference image. IEEE Transactions on Multimedia. 2017;20(8):2049-62.

[7] Oszust M. Local feature descriptor and derivative filters for blind image quality assessment. IEEE Signal Processing Letters. 2019;26(2):322-6.

[8] Leclaire A, Moisan L. No-reference image quality assessment and blind deblurring with sharpness metrics exploiting fourier phase information. Journal of Mathematical Imaging and Vision. 2015;52(1):145-72.

[9] Ding K, Ma K, Wang S, Simoncelli EP. Image quality assessment: Unifying structure and texture similarity. arXiv preprint arXiv:200407728. 2020.

[10] Muthana R, Alshareefi AN, editors. Techniques in De-Blurring Image. Journal of Physics: Conference Series; 2020: IOP Publishing.

[11] Jaramillo Sr BO, Niño-Castañeda JO, Platiša L, Philips W. Content-aware objective video quality assessment. Journal of Electronic Imaging. 2016;25(1):013011.

[12] Kumar A. Deblurring of motion blurred images using histogram of oriented gradients and geometric moments. Signal Processing: Image Communication. 2017;55:55-65.

[13] Kang J, Zhang X, Teng H, Zhao J. Application of maximum correlated Kurtosis deconvolution on bearing fault detection and degradation analysis. Vibroengineering PROCEDIA. 2014;4:119-24.

[14] Han Y, Kan J. Blind Image Deblurring Based on Local Edges Selection. Applied Sciences. 2019;9(16):3274.

[15] Moorthy AK, Bovik AC. Blind image quality assessment: From natural scene statistics to perceptual quality. IEEE transactions on Image Processing. 2011;20(12):3350-64.

[16] Mittal A, Moorthy AK, Bovik AC. No-reference image quality assessment in the spatial domain. IEEE Transactions on image processing. 2012;21(12):4695-708.

[17] Zhang L, Zhang L, Bovik AC. A feature-enriched completely blind image quality evaluator. IEEE Transactions on Image Processing. 2015;24(8):2579-91.

[18] Wang Z, Bovik AC, Sheikh HR, Simoncelli EP. Image quality assessment: from error visibility to structural similarity. IEEE transactions on image processing. 2004;13(4):600-12.

[19] Zhang L, Shen Y, Li H. VSI: A visual saliency-induced index for perceptual image quality assessment. IEEE Transactions on Image processing. 2014;23(10):4270-81.

[20] Li C, Guan T, Zheng Y, Zhong X, Wu X, Bovik A. Blind image quality assessment in the contourlet domain. Signal Processing: Image Communication. 2021;91:116064. 